\title{
Inducción de latencia en semillas desecadas de Scorzonera reverchonii (Compositae) conservadas a baja temperatura
}

\author{
Dormancy induction in dried seeds of Scorzonera reverchonii (Compositae) \\ stored at low temperature
}

\author{
Miguel Ángel Copete ${ }^{\text {a*, Raquel Herranz a }}$, Pablo Ferrandis a, José $\mathbf{M}^{\mathrm{a}}$ Herranz $^{\text {a }}$ \\ * Autor de correspondencia: ${ }^{a}$ Universidad de Castilla-La Mancha, Instituto Botánico, \\ Avda. de la Mancha s/n, 02006, Albacete, España, tel.: +34967599200, miguel.copete@uclm.es
}

\begin{abstract}
SUMMARY
We analyzed the processes of both dormancy break and induction in Scorzonera reverchonii (Compositae) seeds when desiccated to $5 \%$ of humidity and stored at low temperature $\left(5{ }^{\circ} \mathrm{C}\right)$. At dispersal time, seeds were dormant and only $1 \%$ germinated when incubated at $20 / 7{ }^{\circ} \mathrm{C}$ in light/darkness during 30 days. Seeds permanently stored at $5{ }^{\circ} \mathrm{C}$ kept dormant. However, this became viable after two years, since $88-93 \%$ seeds germinated if afterwards they were treated with gibberellic acid. When seeds were stored at room-laboratory conditions (i.e., temperature $=22{ }^{\circ} \mathrm{C}$, relative humidity $=50-60 \%$ ), they gradually surpassed dormancy, and after one year germination reached $85 \%$. Thus, $S$. reverchonii seeds have non-deep physiological dormancy. When seeds stored one year in the room-laboratory (non-dormant seeds) were transferred to $5{ }^{\circ} \mathrm{C}$ in dryness, a gradual induction of secondary dormancy was triggered, reducing germination in 21-29\% after one year in comparison with seeds permanently stored in room-laboratory conditions. After one year at $5{ }^{\circ} \mathrm{C}$, seeds transferred again to room-laboratory germinated progressively, showing germination percentages as high as those in seeds permanently stored in room-laboratory conditions 10 months later.
\end{abstract}

Key words: orthodox seeds, dormancy induction, low temperatures.

\section{RESUMEN}

Se analizó el proceso de rotura e inducción de latencia en semillas de Scorzonera reverchonii (Compositae) desecadas (5 \% de humedad) y conservadas a baja temperatura $\left(5^{\circ} \mathrm{C}\right)$. Las semillas eran latentes en el momento de la dispersión y solo germinaron el $1 \%$ al incubarlas a $20 / 7{ }^{\circ} \mathrm{C}$ luz/oscuridad durante 30 días. Las semillas conservadas de forma permanente a $5{ }^{\circ} \mathrm{C}$ se mantuvieron latentes, pero eran viables transcurridos dos años, ya que germinaron entre $88-93 \%$ al tratarlas con ácido giberélico $\left(\mathrm{GA}_{3}\right)$. Cuando las semillas se conservaron en condiciones de laboratorio $\left(22^{\circ} \mathrm{C}\right.$ y $50-60 \%$ humedad relativa) salieron progresivamente de la latencia y, transcurrido un año, llegaron a germinar el $85 \%$, por lo que su latencia era fisiológica no profunda. Tras un año de conservación en laboratorio (semillas no latentes), las semillas desecadas transferidas a $5{ }^{\circ} \mathrm{C}$ iniciaron un proceso paulatino de inducción de latencia secundaria que, un año después, arrojó porcentajes de germinación entre 21-29 \% inferiores respecto a los de semillas conservadas permanentemente en condiciones de laboratorio. Tras un año de conservación a $5{ }^{\circ} \mathrm{C}$, las semillas transferidas a condiciones de laboratorio germinaron progresivamente $\mathrm{y}$, diez meses después, alcanzaron porcentajes de germinación tan altos como los de las semillas conservadas de forma permanente en condiciones de laboratorio.

Palabras clave: semillas ortodoxas, inducción de latencia, bajas temperaturas.

\section{INTRODUCCIÓN}

La preservación en bancos de semillas constituye la forma más importante de conservación "ex situ" para las especies que poseen semillas de tipo ortodoxo (PérezGarcía et al. 2007). La mayoría de especies de regiones templadas, como la mediterránea, tienen este tipo de semillas (Gómez-Campo 1985, 2006). En la actualidad, las colecciones de los bancos de semillas de especies silvestres complementan a las actividades de conservación "in situ", aportando material genético para llevar a cabo actuaciones de reintroducción o reforzamiento de poblaciones de aquellas especies que lo requieran (Schoen y Brown 2001). El valor e importancia de la conservación "ex situ", como un componente integral de la conservación de la biodiversidad, ha sido reconocido en tratados internacionales, como la Estrategia Mundial para la Conservación de Plantas aprobada en desarrollo del Convenio de Diversidad Biológica, lo que ha propiciado el establecimiento de numerosos bancos de semillas (Cochrane et al. 2007).

Previamente a la conservación de las semillas en un banco es preciso efectuar una prueba de germinación, tan- 
to para evaluar en el tiempo la eficacia del proceso de conservación, como para conocer si el material almacenado tiene problemas de latencia (Gómez-Campo 1985). Así, en 250 especies de diferentes familias conservadas durante 1-26 años en el banco del Jardín Botánico Nacional de Bélgica, Godefroid et al. (2010) encontraron un elevado grado de latencia (germinación $\leq 20 \%$ ). Por ello, estos autores afirmaron que como los requerimientos germinativos de muchas especies raras son poco conocidos, la calidad de una colección de semillas debe ser estimada por su viabilidad y no por el porcentaje de germinación.

Con frecuencia, durante el periodo de conservación las semillas ortodoxas no solo mantienen su viabilidad, sino que pueden superar también la latencia inicial germinando incluso mejor que antes del proceso de conservación. Así, de 37 especies de crucíferas conservadas en condiciones apropiadas para semillas ortodoxas $\left(1,5-3 \%\right.$ humedad y $-5^{\circ} \mathrm{C}$, en tubos de vidrio cerrados a la llama con gel de sílice en el interior) durante 40 años en el banco de germoplasma de la Universidad Politécnica de Madrid, en 35 se mantuvieron o aumentaron los porcentajes de germinación previos al proceso de almacenaje (Pérez-García et al. 2007). Sin embargo, se constató que en las semillas de dos especies (Alyssoides utriculata (L.) Medik. y Matthiola sinuata (L.) R. Br.) se indujo latencia secundaria durante el periodo de conservación. En otro estudio de este mismo grupo de investigación con 15 especies de crucíferas conservadas en las condiciones descritas anteriormente se obtuvieron resultados similares, pero en una especie Diplotaxis harra (Forssk.) Boiss. existió también inducción de latencia durante el almacenaje (Pérez-García et al. 2009).

Aunque la posible inducción de latencia secundaria durante el proceso de conservación de semillas ortodoxas es un fenómeno que no reduce la importancia de los bancos de semillas en las estrategias de conservación "ex situ", debe de ser tenido en cuenta cuando se evalúa la facultad germinativa tras un periodo de conservación.

En este trabajo se evalúa la inducción de latencia en semillas de Scorzonera reverchonii Debeaux ex Hervier (Compositae), especie endémica del sur de la Península Ibérica (cordilleras béticas), con distribución geográfica muy restringida, por lo que ha sido incluida en la Lista Roja de Flora Vascular Española en la categoría Casi Amenazada (Moreno 2008). En ensayos previos se ha comprobado que las semillas de $S$. reverchonii recién dispersadas eran latentes y que las semillas conservadas en cámara frigorífica $\left(5^{\circ} \mathrm{C}\right)$ apenas germinaban o lo hacían peor que las conservadas a temperatura ambiente de laboratorio $\left(22^{\circ} \mathrm{C}\right)$, lo que llevó a plantear este estudio analizando dos poblaciones aisladas entre sí, a fin de verificar que el fenómeno no tiene carácter puntual.

El objetivo general de este trabajo es analizar el proceso paulatino de inducción de latencia en semillas de $S$. reverchonii cuando son desecadas hasta $5 \%$ de humedad y conservadas en cámara frigorífica $\left(5^{\circ} \mathrm{C}\right)$. La hipótesis a verificar es que las semillas que han salido de la latencia tras un periodo de conservación en condiciones de laboratorio, son inducidas a latencia secundaria al transferirlas a $5{ }^{\circ} \mathrm{C}$. Para ello se analiza la evolución durante dos años de la facultad germinativa de estas semillas sometidas a diferentes condiciones de conservación.

\section{MÉTODOS}

Material vegetal, procedencia de las semillas y condiciones de conservación. Scorzonera reverchonii es una planta herbácea vivaz, endémica bética protegida con la categoría de Interés Especial en la región de Castilla-La Mancha (DOCM 2001), donde es más escasa que en Andalucía, su núcleo principal de distribución.

Los frutos (aquenios) de esta especie se recolectaron el 2 de julio de 2014 en dos localidades separadas por $40 \mathrm{~km}$ $\mathrm{y}$, por tanto, aisladas entre sí:

1. Riópar (Albacete, España), a $1.280 \mathrm{~m}$ de altitud, UTM: 30SWH4964, sustrato calizo-dolomítico, en claros de un pinar de Pinus nigra Arnold subsp. salzmannii (Dunal) Franco con Acer granatense Boiss., Cytisus scoparius (L.) Link subsp. reverchonii (Degen et Hervier) Riv. Goday et Riv. Martínez y Echinospartum boissieri (Spach) Rothm.

2. Siles (Jaén, España), a $1.360 \mathrm{~m}$ de altitud, UTM: 30SWH3743, sustrato calizo-dolomítico, en bordes de pinar de Pinus nigra subsp. salzmannii con Quercus faginea Lam. subsp. faginea, Ilex aquifolium L. y Berberis vulgaris L. subsp. australis (Boiss.) Heywood.

De cada población se recolectaron unos 6.000 frutos que se extendieron para que se secaran completamente en bandejas en el laboratorio hasta el 10 de julio de 2014. Tras este periodo, su porcentaje de humedad descendió desde el $8 \%$, en el momento de la recolección, hasta el $5 \%$. Cada cohorte de frutos (en lo sucesivo serán mencionados como semillas) se dividió en cuatro lotes de unas 1.500 semillas cada uno, los lotes se guardaron en frascos de cristal con cierre hermético y se conservaron en las siguientes condiciones:

a) Semillas conservadas de forma permanente en condiciones de laboratorio $\left(22^{\circ} \mathrm{C}\right)$.

b) Semillas conservadas durante un año en condiciones de laboratorio y luego transferidas a $5{ }^{\circ} \mathrm{C}$.

c) Semillas conservadas de forma permanente a $5^{\circ} \mathrm{C}$.

d) Semillas conservadas durante un año a $5{ }^{\circ} \mathrm{C}$ y luego transferidas a condiciones de laboratorio $\left(22^{\circ} \mathrm{C}\right)$.

Ensayos de laboratorio. Los experimentos fueron realizados bajo condiciones de temperatura e iluminación controladas en una cámara de germinación (IBERCEX, modelo $\mathrm{F}-4$, Madrid) con $12 \mathrm{~h}$ de fotoperiodo y un régimen fluctuante de temperaturas de $20 / 7^{\circ} \mathrm{C}$, en el que la temperatura 
más alta coincidía con la fase de iluminación y la más baja con la de oscuridad. Durante la fase de iluminación, las semillas recibieron una iluminancia de 1.250 lux proporcionada por lámparas fluorescentes blancas y frías. Se eligió este régimen de temperatura (habitual de los ambientes mediterráneos durante el otoño y la primavera), porque ha dado buenos resultados en otras especies con similares características ecológicas (Copete et al. 2005), y tras comprobar su idoneidad en ensayos preliminares efectuados durante 2013.

Las semillas de las dos poblaciones estudiadas se colocaron en placas Petri de $9 \mathrm{~cm}$ de diámetro sobre dos discos de papel de filtro humedecidos con agua destilada. Las placas se sellaron con parafilm para reducir la pérdida de agua y se utilizaron para cada ensayo cuatro réplicas de 25 semillas cada una.

Para cada una de las condiciones de conservación de las semillas se efectuaron ensayos de germinación con periodicidad bimensual, desde el 14 de julio de 2014 hasta el 1 de julio de 2016, con la excepción de las semillas sometidas a las condiciones de conservación b y d, en las que los ensayos se iniciaron el 1 de septiembre de 2015. El 1 de julio de 2016, con la finalidad de comprobar que las semillas conservadas de forma permanente a $5^{\circ} \mathrm{C}$ eran latentes y viables, se efectuó un ensayo adicional incubando dichas semillas a las mismas temperaturas en una solución de $1.000 \mathrm{mg} \mathrm{L}^{-1}$ de ácido giberélico $\left(\mathrm{GA}_{3}\right)$.

La duración de los ensayos de germinación fue de 30 días (Baskin y Baskin 2014), efectuando el control de la germinación cada dos o tres días, momento en el que se anotaban y retiraban de las placas las semillas germinadas (semillas con radícula $\geq 1 \mathrm{~mm}$ ).

Al final de cada ensayo se abrieron, con ayuda de pinzas y bisturí, las semillas no germinadas, para comprobar si el embrión tenía un aspecto blanquecino y era turgente (semilla viable) o si era marrón oscuro y blando (semilla inviable). En cada réplica el porcentaje de germinación se calculó sobre el número total de semillas viables.

Tratamiento estadístico. La respuesta germinativa se evaluó mediante el análisis de dos parámetros: 1) el porcentaje final de germinación sobre semillas viables; y 2) la velocidad de germinación medida por el parámetro $\mathrm{T}_{50}$, que se define como el tiempo preciso (expresado en días) para lograr la mitad del porcentaje final de germinación (Thanos y Georghiou 1988). El parámetro $\mathrm{T}_{50}$ solo se calculó para los ensayos con germinación final $\geq 10 \%$, ya que valores inferiores no se consideran representativos.

La evaluación del efecto de las diferentes condiciones de conservación y la edad de las semillas sobre el porcentaje final y velocidad de germinación se realizó mediante un ANDEVA de dos factores a fin de detectar diferencias significativas. Los casos responsables de efectos principales significativos se detectaron mediante una prueba múltiple de Tukey. Previamente a la realización del análisis, las condiciones de normalidad (prueba de Cochran) y homocedasticidad (prueba de David) de los datos fueron comprobadas (Zar 2010). Los porcentajes de germinación fueron transformados para su análisis a la raíz cuadrada de su arcoseno, a fin de ajustar su distribución a la normal. En la figura estos porcentajes se representan sin transformar. Esta transformación no fue necesaria para el $\mathrm{T}_{50}$.

Los resultados obtenidos se evaluaron independientemente para cada una de las dos poblaciones estudiadas.

\section{RESULTADOS}

Facultad germinativa. Las semillas recién dispersadas (julio 2014) eran latentes y solo germinó el $1 \%$, tanto en la población de Riópar como en la de Siles. Sin embargo, cuando las semillas se conservaron en condiciones de laboratorio salieron paulatinamente de la latencia y en el ensayo de mayo de 2015 (10 meses de conservación) se alcanzó una germinación final del 69 \% en la población de Riópar y un $51 \%$ en la de Siles. En la población de Riópar los valores más altos de germinación (88 \%) se alcanzaron en julio de 2015 (12 meses de conservación) y, a partir de este momento, los porcentajes de germinación presentaron pequeñas fluctuaciones mensuales con desviaciones poco significativas. En la población de Siles el incremento de los porcentajes de germinación en el curso del tiempo fue más lento y los porcentajes más altos $(84 \%)$ se alcanzaron en julio de 2016, al final del estudio, presentando también pequeñas fluctuaciones la evolución de dicho porcentajes (figura 1).

Las semillas conservadas de forma permanente a $5{ }^{\circ} \mathrm{C}$ no salieron de la latencia a lo largo de los dos años de duración del estudio y los porcentajes de germinación alcanzados oscilaron entre 0-2 \% para ambas poblaciones (figura 1).

Cuando tras un año de conservación en condiciones de laboratorio (semillas no latentes en la población de Riópar y parcialmente latentes en la de Siles) las semillas fueron transferidas a $5{ }^{\circ} \mathrm{C}$ empezaron a disminuir de forma progresiva los porcentajes de germinación en relación con los de las semillas conservadas a temperatura de laboratorio $\left(22{ }^{\circ} \mathrm{C}\right)$, de forma que fueron significativamente inferiores en julio de 2016 (12 meses después) en la población de Riópar y a partir de marzo de 2016 (ocho meses después) en la población de Siles, llegando a ser entre un 21-29\% inferiores al final del estudio (figura 1).

Cuando tras un año de conservación en cámara frigorífica a $5{ }^{\circ} \mathrm{C}$ las semillas fueron transferidas a temperatura de laboratorio empezó un proceso paulatino de salida de la latencia, de forma que en el ensayo iniciado en enero de 2016 (seis meses después) se alcanzó el $44 \%$ de germinación en la población de Riópar y el 27 \% en la de Siles. A partir de este momento, el incremento de los porcentajes de germinación fue continuo, de forma que en los ensayos iniciados en mayo de 2016 y julio de 2016 se alcanzaron valores por encima del $80 \%$ en ambas poblaciones (figura 1). 
En el ensayo con ácido giberélico $\left(\mathrm{GA}_{3}\right)$ iniciado en julio de 2016 con semillas conservadas de forma permanente a $5{ }^{\circ} \mathrm{C}$ hasta ese momento, se alcanzó una germinación del $93 \%$ en la población de Riópar y un $89 \%$ en la de Siles (datos no representados en la figura).

En los diferentes ensayos de germinación realizados, el porcentaje de semillas inviables osciló entre 0-5\%.

Velocidad de germinación. En las semillas conservadas en condiciones de laboratorio el proceso de salida de la latencia y aumento progresivo de los porcentajes de germinación fue acompañado también de un aumento de la velocidad de germinación, con disminución de los valores del parámetro $\mathrm{T}_{50}$. Sin embargo, esta disminución no fue significativa en la población de Riópar, y solo fue significativa a partir de los 22 meses de conservación en la población de Siles (cuadro 1).

Cuando las semillas conservadas durante un año en condiciones de laboratorio fueron transferidas a $5{ }^{\circ} \mathrm{C}$, su velocidad de germinación se ralentizó (valores más altos
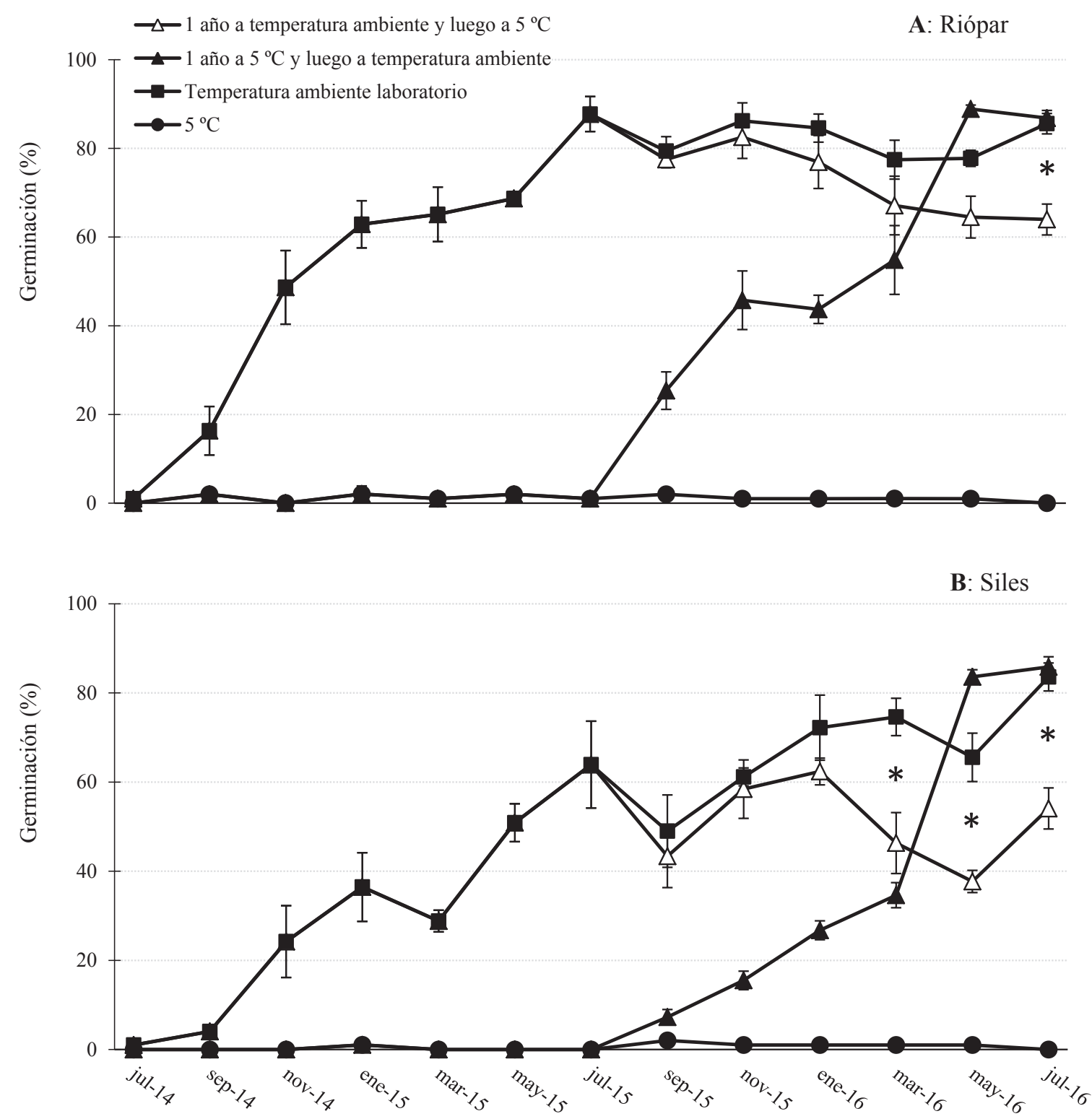

Mes y año

Figura 1. Evolución de los porcentajes de germinación de semillas de Scorzonera reverchonii, de las poblaciones de Riópar (A) y Siles (B), conservadas durante dos años bajo diferentes condiciones. El símbolo* denota las fechas en las que las diferencias de germinación entre las condiciones - $-\mathrm{y}-\Delta$ - son significativas $(P<0,05)$.

Changes in the germination percentages in Scorzonera reverchonii seeds coming from Riópar (A) and Siles (B), stored at different conditions for two years. Asterisks denote those dates where differences in germination between $-\mathbf{m}-$ and $-\Delta-$ treatments were significant $(P<0.05)$. 
Cuadro 1. Velocidad de germinación $\left(\mathrm{T}_{50}\right.$ en días, media $\pm \mathrm{SE}$, germinación final $\left.\geq 10 \%\right)$ de semillas de Scorzonera reverchonii conservadas bajo diferentes condiciones durante dos años. A, semillas conservadas de forma permanente en condiciones de laboratorio $\left(22^{\circ} \mathrm{C}\right)$; B, semillas conservadas un año en condiciones de laboratorio y luego transferidas a $5{ }^{\circ} \mathrm{C}$; y $\mathrm{C}$, semillas conservadas durante un año a $5{ }^{\circ} \mathrm{C}$ y luego transferidas a condiciones de laboratorio $\left(22^{\circ} \mathrm{C}\right)$. Letras mayúsculas diferentes dentro de la misma columna y, para cada población, letras minúsculas diferentes en la misma fila indican diferencias significativas $(P<0,05)$.

Germination rate $\left(\mathrm{T}_{50}\right.$ in days, mean \pm standard error, germination $\left.\geq 10 \%\right)$ in seeds of Scorzonera reverchonii stored at different conditions for two years. A) seeds permanently kept at laboratory conditions $\left(22^{\circ} \mathrm{C}\right)$; B) seeds stored during one year in the laboratory and subsequently transferred to $5^{\circ} \mathrm{C}$; C) seeds stored one year at $5^{\circ} \mathrm{C}$ and afterward transferred at laboratory conditions $\left(22^{\circ} \mathrm{C}\right)$. Different capital letters within a column and different lowercase letters within a row denote significant differences between treatments in a population $(P<0.05)$.

\begin{tabular}{|c|c|c|c|c|c|c|}
\hline \multirow{4}{*}{$\begin{array}{l}\text { Fecha ensayo de } \\
\text { germinación }\end{array}$} & \multicolumn{6}{|c|}{ Población } \\
\hline & \multicolumn{3}{|c|}{ Riópar } & \multicolumn{3}{|c|}{ Siles } \\
\hline & \multicolumn{3}{|c|}{ Condición de conservación } & \multicolumn{3}{|c|}{ Condición de conservación } \\
\hline & A & B & $\mathrm{C}$ & A & B & $\mathrm{C}$ \\
\hline Septiembre 2014 & $21,5 \pm 2,3^{\mathrm{ABa}}$ & $21,5 \pm 2,3^{\mathrm{ABa}}$ & - & - & - & - \\
\hline Noviembre 2014 & $25,3 \pm 0,7^{\mathrm{Ba}}$ & $25,3 \pm 0,7^{\mathrm{Ba}}$ & - & $27,5 \pm 0,8^{\mathrm{Ca}}$ & $27,5 \pm 0,8^{\mathrm{Ca}}$ & - \\
\hline Enero 2015 & $18,3 \pm 1,9^{\mathrm{ABa}}$ & $18,3 \pm 1,9^{\mathrm{ABa}}$ & - & $25,0 \pm 1,1^{\mathrm{BCa}}$ & $25,0 \pm 1,1^{\mathrm{BCa}}$ & - \\
\hline Marzo 2015 & $17,0 \pm 1,7^{\mathrm{Aa}}$ & $17,0 \pm 1,7^{\mathrm{ABa}}$ & - & $24,5 \pm 0,8^{\mathrm{BCa}}$ & $24,5 \pm 0,8^{\mathrm{BCa}}$ & - \\
\hline Mayo 2015 & $17,5 \pm 1,3^{\mathrm{ABa}}$ & $17,5 \pm 1,3^{\mathrm{ABa}}$ & - & $20,8 \pm 2,0^{\mathrm{ABCa}}$ & $20,8 \pm 2,0^{\mathrm{ABa}}$ & - \\
\hline Julio 2015 & $21,0 \pm 2,0^{\mathrm{ABa}}$ & $21,0 \pm 2,0^{\mathrm{ABa}}$ & - & $23,3 \pm 1,5^{\mathrm{ABCa}}$ & $23,3 \pm 1,5^{\mathrm{BCa}}$ & - \\
\hline Septiembre 2015 & $14,5 \pm 0,7^{\text {Aa }}$ & $15,3 \pm 0,7^{\text {Аа }}$ & $23,5 \pm 2,5^{\mathrm{BCb}}$ & $24,3 \pm 3,3^{\mathrm{ABCa}}$ & $28,0 \pm 0^{\mathrm{Ca}}$ & - \\
\hline Noviembre 2015 & $17,5 \pm 1,8^{\mathrm{ABa}}$ & $15,3 \pm 2,0^{\mathrm{Aa}}$ & $23,0 \pm 1,7^{\mathrm{ABCa}}$ & $22,0 \pm 2,1^{\mathrm{ABCa}}$ & $18,3 \pm 2,0^{\mathrm{Aa}}$ & $24,3 \pm 3,9^{\mathrm{Aa}}$ \\
\hline Enero 2016 & $16,5 \pm 0,4^{\mathrm{Aa}}$ & $17,5 \pm 0,8^{\mathrm{ABa}}$ & $24,8 \pm 1,1^{\mathrm{Cb}}$ & $18,3 \pm 0,7^{\mathrm{ABa}}$ & $24,0 \pm 0^{\mathrm{BCa}}$ & $23,0 \pm 2,7^{\text {Aa }}$ \\
\hline Marzo 2016 & $15,0 \pm 0,5^{\mathrm{Aa}}$ & $20,8 \pm 1,5^{\mathrm{ABb}}$ & $24,0 \pm 1,2^{\mathrm{Cb}}$ & $21,0 \pm 0^{\mathrm{ABCa}}$ & $23,3 \pm 0,7^{\mathrm{BCa}}$ & $23,5 \pm 1,3^{\text {Aa }}$ \\
\hline Mayo 2016 & $13,8 \pm 1,2^{\mathrm{Aa}}$ & $21,0 \pm 0^{\mathrm{ABb}}$ & $16,0 \pm 0^{\mathrm{Aa}}$ & $15,5 \pm 0,8^{\mathrm{Aa}}$ & $20,5 \pm 1,8^{\mathrm{ABa}}$ & $16,0 \pm 0^{\mathrm{Aa}}$ \\
\hline Julio 2016 & $13,8 \pm 0,7^{\text {Аа }}$ & $13,8 \pm 0,7^{\mathrm{Aa}}$ & $16,5 \pm 0,4^{\mathrm{ABb}}$ & $16,5 \pm 1,8^{\mathrm{ABa}}$ & $20,0 \pm 0^{\mathrm{ABa}}$ & $16,5 \pm 0,4^{\text {Аa }}$ \\
\hline
\end{tabular}

del parámetro $\mathrm{T}_{50}$ ) a partir de marzo de 2016 (ocho meses de conservación), pero las diferencias solo llegaron a ser significativas en la población de Riópar (cuadro 1). Cuando las semillas conservadas durante un año a $5{ }^{\circ} \mathrm{C}$ fueron transferidas a temperatura de laboratorio, la velocidad de germinación fue más lenta durante los ocho meses siguientes y, al igual que antes, las diferencias solo fueron significativas en la población de Riópar (cuadro 1).

\section{DISCUSIÓN Y CONCLUSIONES}

Las semillas de $S$. reverchonii presentan latencia fisiológica ya que tienen embriones desarrollados en el momento de la dispersión y, además, embeben agua sin dificultad cuando se incuban sobre papel de filtro humedecido. Por otra parte, las semillas desecadas salen paulatinamente de la latencia a partir de dos meses de conservación en condiciones de laboratorio y, además, el ácido giberélico $\left(\mathrm{GA}_{3}\right)$ estimula su germinación, por lo que se puede afirmar que el nivel de latencia fisiológica presente es no profundo (según el criterio de Baskin y Baskin 2014).

Cuando semillas desecadas que han salido de la latencia tras un año de conservación a temperatura ambiente de laboratorio se transfieren a $5{ }^{\circ} \mathrm{C}$, los porcentajes de germinación empiezan a disminuir siendo estadísticamente significativo el descenso a partir de los ocho meses en la población de Siles (figura 1B) y a partir de los 12 en la de Riópar (figura 1A). Por lo tanto, en ambas poblaciones puede hablarse de inducción de latencia secundaria en sus semillas y, de esta forma, los resultados obtenidos en este estudio confirman la hipótesis de partida. Es conocida la inducción de latencia secundaria en numerosas especies con latencia fisiológica no profunda, y que son anuales de invierno obligadas o facultativas, cuando sus semillas hidratadas (35-45\% humedad) son expuestas a temperaturas de estratificación fría $\left(5^{\circ} \mathrm{C}\right)$ como las empleadas en este estudio. Así ocurre, en Veronica arvensis L. (Baskin y Baskin 1986), Stellaria media (L.) Vill., Arenaria serpyllifolia L. y Cerastium viscosum L. (Baskin y Baskin 1986), y en Iberis pectinata Boiss. y Ziziphora aragonensis Pau (Copete et al. 2009), entre otras. También son inducidas a latencia secundaria por bajas temperaturas semillas hidratadas de especies de matorral como Sideritis serrata Lag. (Copete et al. 2015) y de varios geófitos con latencia morfofisiológica simple y profunda del epicótilo, como ocurre en Narcissus hispanicus Gouan (Copete et al. 2011), 
N. eugeniae Fern. Casas (Copete et al. 2014) y N. radinganorum Fern. Casas (Herranz et al. 2015). Sin embargo, la inducción de latencia secundaria cuando las semillas se conservan en seco a baja temperatura es un fenómeno poco conocido en la bibliografía, como los casos de Alyssoides utriculata y Matthiola sinuata (Pérez-García et al. 2007) y Diplotaxis harra (Pérez-García et al. 2009).

La facultad germinativa de las semillas de ambas poblaciones conservadas de forma permanente en condiciones de laboratorio presentó pequeñas fluctuaciones mensuales. Algo similar ocurrió con las semillas transferidas a $5{ }^{\circ} \mathrm{C}$ después de un año de conservación en condiciones de laboratorio, con fluctuaciones más acusadas y estadísticamente significativas en la población de Siles. Fluctuaciones similares se han encontrado en semillas de Sisymbrium runcinatum Lag. ex DC. (Herranz et al. 2003), así como en otras crucíferas como Diplotaxis erucoides (L.) DC. y D. virgata (Cav.) DC. (Pérez-García et al. 1995). Gutterman (1994) también describió oscilaciones mensuales en la germinación de semillas de Mesembryanthemum nodiflorum L., refiriéndose al fenómeno como ciclos endógenos de latencia.

Las dos poblaciones estudiadas muestran un modelo germinativo parecido y las diferentes gráficas de la figura 1 presentan la misma tendencia. Sin embargo, hay diferencias evidentes entre ambas poblaciones; las semillas de la población de Siles presentan porcentajes iniciales de germinación más bajos, tardando más tiempo en superar la latencia, y tienen velocidades iniciales de germinación más lentas. La variabilidad interpoblacional en el grado de latencia es un fenómeno muy conocido (Andersson y Milberg 1998), pudiendo ser considerada de carácter genotípico, y representando una adaptación al hábitat regional (Meyer 1992). También puede ser el resultado de las condiciones ambientales de cada sitio durante el desarrollo y la maduración de las semillas, el denominado efecto materno (Fenner 1991).

Aunque muchos bancos de semillas de especies cultivadas utilizan para sus colecciones base (a largo plazo) temperaturas de conservación ultrabajas (entre -10 y $-18{ }^{\circ} \mathrm{C}$ ), se pueden obtener buenos resultados sin necesidad de recurrir a temperaturas tan bajas, dado su alto coste energético. De hecho, Gómez-Campo (1985) indica que puede alargarse considerablemente la vida de las semillas ortodoxas si estas se desecan previamente (sin sobrepasar el límite inferior del 1-3\% en su contenido de humedad) y después se conservan en recipientes herméticos a la temperatura de un refrigerador $\left(0-5^{\circ} \mathrm{C}\right)$. Por lo tanto, algunas de las condiciones de conservación evaluadas en este estudio $\left(5 \%\right.$ humedad de semillas, $\left.5{ }^{\circ} \mathrm{C}\right)$ pueden considerarse adecuadas para conservación de semillas ortodoxas. Sin embargo, tras un periodo de dos años las semillas siguen en estado de latencia (pero viables, ya que germinan con $\mathrm{GA}_{3}$ ) o pueden ser inducidas a un estado de latencia secundaria cuando, tras un año a temperatura ambiente de laboratorio, se transfieren a $5{ }^{\circ} \mathrm{C}$.
Aunque habitualmente el proceso de preservación de semillas ortodoxas garantiza el mantenimiento de su viabilidad y la posterior germinación (Pérez-García et al. 2007, 2008), deben tenerse en cuenta tanto el posible mantenimiento de las semillas en un estado de latencia fisiológico primario, como la probable inducción de latencia secundaria, especialmente cuando se trate de hacer germinar a las semillas para producir planta.

\section{AGRADECIMIENTOS}

A los revisores del artículo por las sugerencias realizadas, que han permitido facilitar su comprensión y que mejore apreciablemente la calidad del mismo.

\section{REFERENCIAS}

Andersson L, P Milberg. 1998. Variation in seed dormancy among mother plants, populations and years of seed collection. Seed Science Research 8:29-38.

Baskin CC, JM Baskin. 2014. Seeds. Ecology, biogeography and evolution of dormancy and germination. $2^{\mathrm{a}}$ ed. San Diego, California, USA. Academic Press. 1573 p.

Baskin JM, CC Baskin. 1986. Temperature requirements for after-ripening in seeds of nine winter annuals. Weed Research 26:375-380.

Cochrane JA, AD Crawford, LT Monks. 2007. The significance of ex-situ seed conservation to reintroduction of threatened plants. Australian Journal of Botany 55:356-361.

Copete E, JM Herranz, MA Copete, P Ferrandis. 2014. Interpopulation variability on embryo growth, seed dormancy break, and germination in the endangered Iberian daffodil Narcissus eugeniae (Amaryllidaceae). Plant Species Biology 29:e72-e84.

Copete E, JM Herranz, P Ferrandis, CC Baskin, JM Baskin. 2011. Physiology, morphology and phenology of seed dormancy-break and germination in the endemic Iberian species Narcissus hispanicus (Amaryllidaceae). Annals of Botany 107:1003-1016.

Copete MA, JM Herranz, P Ferrandis. 2005. Seed dormancy and germination in threatened Iberian Coincya (Brassicaceae) taxa. Écoscience 12(2):257-266.

Copete MA, JM Herranz, P Ferrandis. 2009. Seed germination ecology of the endemic Iberian winter annuals Iberis pectinata and Ziziphora aragonensis. Seed Science Research 19:155-169.

Copete MA, JM Herranz, P Ferrandis, E Copete. 2015. Annual dormancy cycles in buried seeds of shrub species: germination ecology of Sideritis serrata (Labiatae). Plant Biology 17:798-807.

DOCM. 2001. Decreto 200/2001, de 06-11-2001, por el que se modifica el Catálogo Regional de Especies Amenazadas. Diario Oficial de Castilla-La Mancha 119:12825-12827.

Fenner M. 1991. The effects of the parent environment on seed germinability. Seed Science Research 1:75-84.

Godefroid S, A Van de Vyver, T Vanderborght. 2010. Germination capacity and viability of threatened species collections in seed banks. Biodiversity and Conservation 19:13651383 . 
Gómez-Campo C. 1985. Seed banks as an emergency conservation strategy. In Gómez-Campo C ed. Plant Conservation in the Mediterranean Area. Dordrecht, The Netherlands. Junk Publishers. p. 237-247.

Gómez-Campo C. 2006. Erosion of genetic resources within seed genebanks: the role of seed containers. Seed Science Research 16:291-294.

Gutterman Y. 1994. Strategies of seed dispersal and germination in plants inhabiting deserts. Botanical Review 60:373-425.

Herranz JM, E Copete, MA Copete, P Ferrandis. 2015. Germination ecology of the endemic Iberian daffodil Narcissus radinganorum (Amaryllidaceae). Dormancy induction by cold stratification or desiccation in late stages of embryo growth. Forest Systems 24(1):e013.

Herranz JM, P Ferrandis, MA Copete. 2003. Influence of light and temperature on seed germination and ability of the endangered plant species Sisymbrium cavanillesianum to form persistent soil seed banks. Écoscience 10(4):532-541.

Meyer SE. 1992. Habitat-correlated variation in firecracker penstemon (Penstemon eatonii: Scrophulariaceae) seed germination patterns. Bulletin of the Torrey Botanical Club 119:268-279.

Moreno JC (coord.). 2008. Lista Roja 2008 de la Flora Vascular Española. Madrid, España. Dirección General de Medio Natural y Política Forestal. Ministerio de Medio Ambiente y Medio Rural y Marino - Sociedad Española de Biología de la Conservación de las Plantas. 86 p.

Pérez-García F, C Gómez-Campo, RH Ellis. 2009. Successful long-term ultra dry storage of seeds of 15 species of Brassicaceae in a genebank: variation in ability to germinate over 40 years and dormancy. Seed Science and Technology 37:640-649.

Pérez-García F, ME González-Benito, C Gómez-Campo. 2007. High viability recorded in ultra-dry seeds of 37 species of Brassicaceae after almost 40 years of storage. Seed Science and Technology 35:143-153.

Pérez-García F, ME González-Benito, C Gómez-Campo. 2008. Germination of fourteen endemic species from the Iberian Peninsula, Canary and Balearic Islands after 32-34 years of storage at low temperature and very low water content. Seed Science and Technology 36:407-422.

Pérez-García F, JM Iriondo, JB Martínez-Laborde. 1995. Germination behaviour in seed of Diplotaxis erucoides and D. virgata. Weed Research 35:495-502.

Schoen DJ, AH Brown. 2001. The conservation of wild plant species in seed banks. BioScience 51(11):960-966.

Thanos CA, K Georghion. 1988. Ecophysiology of fire stimulated seed germination in Cistus incanus subsp. creticus (L.) Heywood and C. salvifolius L. Plant, Cell, and Environment 11:841-849.

Zar JH. 2010. Biostatistical analysis. 5th ed. Upper Saddle River, USA. Prentice Hall. 944 p.

Recibido: 29.09 .17

Aceptado: 01.03.18 
